\section{COMUNIDADE DE PEQUENOS MAMÍFEROS NÃO \\ VOADORES E SUA ASSOCIAÇÃO COM ESTRUTURAS
FITOFISIONOMICAS DISTINTAS NA SERRA DO \\ VOADORES E SUA ASSOCIAÇÃO COM ESTRUTURA
FITOFISIONOMICAS DISTINTAS NA SERRA DO LIVRAMENTO, PERNAMBUCO, BRASIL}

RESUMO

As relações entre as comunidades de pequenos mamíferos em habitats fitofisionomicamente distintos foram examinadas em três áreas distintas na Serra do Livramento no sertão Pernambucano. O estudo foi realizado no período de dezembro/2013 a maio/2014 e buscou contribuir para o conhecimento da composição da comunidade de pequenos mamíferos dessa área, bem como correlacioná-la com a estrutura do habitat. Os pequenos mamíferos foram capturados com auxilio de armadilhas de queda dispostas em três grades de amostragens. Thrichomys apereoides foi a espécie mais abundante e com maior frequência na grade I. Monodelphis domestica e Gracilinanus agilis foram exclusivos na grade I, enquanto de Calomys exupulsus foi exclusivo na grade III. O índice de similaridade de Morisita indicou elevada similaridade entre as grades I e II e baixa entre as grades I e III. Os parâmetros fitossociológicos como densidade, dominância, altura média e a ocorrência de algumas famílias botânicas contribuem de diferentes maneiras para a ocorrência das diferentes espécies de pequenos mamíferos nos habitats amostrados. Os achados desse estudo demonstram a importância dos estudos com pequenos mamíferos em diferentes Caatingas para uma melhor compreensão dos efeitos das pressões antropogênicas sobre os diferentes grupos faunísticos.

PALAVRAS-CHAVES: Caatinga; Semiárido; Marsupiais; Roedores.

\section{COMMUNITY OF SMALL NON-FLYING MAMMALS AND THEIR ASSOCIATION WITH DIFFERENT PHYTOPHYSIONOMIC STRUCTURES IN THE SALVATION OF THE MOUNTAINS, PERNAMBUCO, BRAZIL}

\section{ABSTRACT}

The relationships between small mammals community structure in different habitat phitophysionomy was examined on three áreas in the Serra do Livramento, semiarid the of Pernambuco State. Data on the small mammals were obtained throught a mark-recapture trapping program from December 2013 to May 2014 and aiming to contribute to the knowledge of small mammals community composition in this area and correlate with the habitat structure. Thrichomys apereoides was most abundant and more frequently in area I. Monodelphis domestica and Gracilinanus agilis occurred only in area I, while Calomys exupulsus was unique in area III. Morisita Similarity Index indicated high similarity between areas I and II, low and similarity between areas I and III. Phytosociological parameters such as density, predominance, height and botanical families contribute in different ways to the occurrence of different small mammals species in the sampled habitats. These findings corroborate the importance of small mammals studies on areas with anthropogenic pressure in the Caatinga.

KEYWORDS: Caatinga; Semiarid; Marsupials; Rodents.
Revista Ibero-Americana de

Ciências Ambientais, Aquidabã, v.6, n.1, Dez 2014, Jan, Fev, Mar, Abr, Mai 2015.

ISSN 2179-6858

\section{SECTION: Articles} TOPIC: Notas Científicas

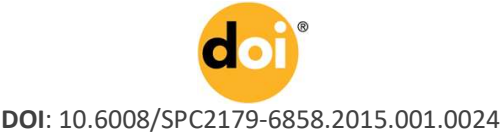

Daniele Santana Sampaio

Universidade Federal do Vale do São Francisco, Brasi http://lattes.cnpq.br/1397296419756212 danydesantana@yahoo.com.br

lardley Cícero Gomes Varjão Universidade Federal do Vale do São Francisco, Brasil http://lattes.cnpq.br/7166654568213057 iardleyvarjao@hotmail.com

Patricia Avello Nicola

Universidade Federal do Vale do São Francisco, Brasil http://lattes.cnpq.br/2526394160477046 patricia.nicola@univasf.edu.br

Received: 02/09/2014

Approved: $14 / 10 / 2015$

Reviewed anonymously in the process of blind peer.

Referencing this:

SAMPAIO, D. S.; VARJÃO, I. C. G.; NICOLA, P. A. Comunidade de pequenos mamíferos não voadores $e$ sua associação com estruturas fitofisionomicas distintas na serra do livramento, Pernambuco, Brasil. Revista Ibero-Americana de Ciências Ambientais, Aquidabã, v.6, n.1, p.307-322, 2015. DOI: http://dx.doi.org/10.6008/SPC21796858.2015 .001 .0024 


\section{INTRODUÇÃO}

A Caatinga é um dos domínios morfoclimáticos mais severamente explorados, promovendo um acelerado processo de degradação (GARIGLIO et al., 2010). Portanto, a manutenção da alta riqueza de espécies e do grau elevado de endemismo da Caatinga (TABARELLI \& SILVA, 2003) depende atualmente de processos de estratégias de conservação para tentar minimizar a perda da biodiversidade deste bioma. Nesse contexto, é relevante a realização de estudos sobre as comunidades de pequenos mamíferos não voadores, principalmente porque nas Caatingas ainda existem lacunas de conhecimento sobre o padrão de distribuição e o uso do habitat por pequenos mamíferos.

Apesar de o Brasil representar o país que abriga o maior número de espécies de mamíferos do mundo, existem lacunas de conhecimento sobre muitos aspectos básicos tais como a distribuição geográfica, taxonomia e o uso do habitat, especialmente entre os pequenos mamíferos, pois não são frequentes os estudos que relacionam a diversidade com a heterogeneidade em áreas de (FREITAS et al., 2005). Diferentes espécies de pequenos mamíferos são usualmente utilizadas para determinar o padrão de distribuição e uso do habitat em ambientes com gradientes de heterogeneidade estrutural, devido a sua relativa facilidade de captura e por se distribuírem vertical e horizontalmente no ambiente (VIVEIROS DE CASTRO \& FERNANDEZ 2004; REVILLA et al., 2004; FOREIRO-MEDINA \& VIEIRA, 2007; REVILLA; WIEGAND, 2008).

O objetivo geral deste estudo foi verificar a diversidade de pequenos mamíferos não voadores na Serra do Livramento, no estado de Pernambuco, Brasil, tendo como objetivos específicos: estimar a riqueza e a diversidade de espécies de pequenos mamíferos na Serra do Livramento; comparar riqueza e a diversidade de espécies de pequenos mamíferos em três diferentes habitats; calcular e comparar a similaridade das comunidades de pequenos mamíferos em três diferentes habitats; relacionar os parâmetros fitossociológicos com a ocorrência das espécies de pequenos mamíferos em cada um dos três diferentes habitats estudados.

\section{MATERIAIS E MÉTODOS}

\section{Relato}

O estudo da comunidade de pequenos mamíferos não voadores foi realizado na Serra do Livramento, municípios de Salgueiro e Cabrobó, no estado de Pernambuco, Brasil (39¹7'31' L e $8^{\circ} 12$ '48" O). Para a coleta de dados da comunidade vegetal e de pequenos mamíferos foram instaladas três grades de amostragens na Serra do Livramento. Na grade de amostragem I foram registradas 18 espécies e 11 famílias (Apendice A). Euphorbiaceae (5) e Cactaceae (3) foram as mais ricas. Croton blanchetianus (Euphorbiaceae) e Erytroxylum sp. (Erytroxylaceae) destacaramse como as mais importantes, principalmente pelos índices de valor de cobertura (IVC) $(49,2 \%$ e 
11,19\%, respectivamente). Croton blanchetianus (Euphorbiaceae), Erytroxylum sp. (Erytroxylaceae) e Myracrodruon urundeuva (Anacardiaceae) apresentam os maiores valores de frequência relativa. A altura média observada para a grade I foi de 3,73 $\mathrm{m}$.

$\mathrm{Na}$ grade de amostragem II foram registradas 18 espécies e oito famílias. Euphorbiaceae (6) foi a família mais rica seguida de Fabaceae (3) e Anacardiaceae (3) (Apêndice A). Croton heliotropiifolius (Euphorbiaceae), Amburana cearensis (Fabaceae), Spondias tuberosa (Anacardiaceae) e Cnidoscolus quercifolius (Euphorbiaceae) foram as apresentaram os maiores índices de valores de cobertura $(33,31 \%, 14,9 \%, 11,97 \%$ e $10,29 \%$, respectivamente). Croton heliotropiifolius (Euphorbiaceae), Cnidoscolus quercifolius (Euphorbiaceae) e Jatropha molissima (Euphorbiaceae) foram as espécies que apresentaram a maior frequência relativa na grade II (12,5\% cada). As espécies dominantes na grade II foram Spondias tuberosa (Anacardiaceae), Croton heliotropiifolius (Euphorbiaceae), Commiphora leptophloeos (Burseraceae) e Anadenanthera macrocarpa (Fabaceae). Foram registradas para a grade de amostragem III, 22 espécies e nove famílias. Fabaceae (5) e Euphorbiaceae (5) foram as mais ricas, compreendendo $45,45 \%$ das espécies desta área. Diferente das demais áreas, na grade III, somente Croton heliotropiifolius (Euphorbiaceae) destacou-se como um índice de valor de cobertura elevado, $31,2 \%$. As espécies Croton heliotropiifolius (Euphorbiaceae), Myracrodruon urundeuva (Anacardiaceae) e Poincianella pyramidalis (Fabaceae) dominaram a paisagem da grade III, que apresentou a menor altura média $(2,93 \mathrm{~m})$ entre as três áreas estudadas.

Através da análise de agrupamentos (UPGMA), elaborada com base no levantamento fitossociológico, foram evidenciados dois grupos de comunidades vegetais. Um dos grupos reúne as comunidades estabelecidas na grade I (parcelas 1 a 5) e o segundo grupo reúne as comunidades estabelecidas na grade II (parcelas 6 a 10). A grade III mostrou apresentar porções similares a fitofisionomia encontrada na grade I (parcelas 11 e 12) e porções similares a fitofisionomia da grade II (parcelas 13, 14 e 15), o que contribuem no entendimento da maior diversidade de espécies observada na grade III (Gráfico 1).

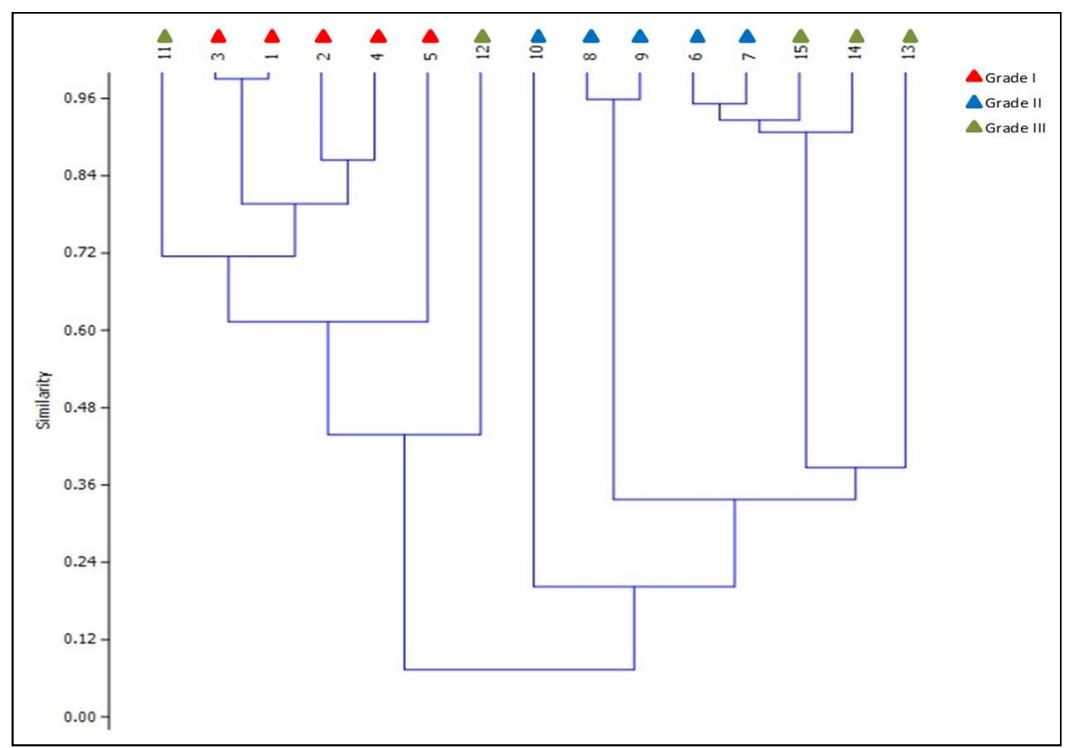

Gráfico 1: Dendograma de similaridade de Morisita entre as parcelas das grades de amostragens. 
Cada grade de amostragem continha cinco transectos espaçados entre si em 100m e em cada transecto foram instaladas oito estações fixas de captura de pequenos mamíferos, espaçadas entre si em 50m, formando uma grade de captura com área total de 14 ha. Em cada grade foram utilizadas 20 armadilhas do tipo Sherman live trap com dimensões de $31 \mathrm{~cm} \times 8,5 \mathrm{~cm} \times$ $11 \mathrm{~cm}$ e as armadilhas do tipo Tomahawk live trap com dimensões de $51 \mathrm{~cm} \times 20 \mathrm{~cm} \times 23,5 \mathrm{~cm}$, distribuídas de forma intercalada, totalizando 40 armadilhas por grade de captura (Gráfico 2).

Mensalmente e durante cinco noites consecutivas, entre dezembro/2013 a maio/2014, as armadilhas foram armadas com iscas de banana e pasta de amendoim, misturadas com óleo de fígado de bacalhau (PAGLIA et al., 1995; CÁCERES \& MONTEIRO-FILHO, 1998), sendo diariamente vistoriadas entre 06:00 e 09:30 horas, re-iscadas quando necessário ou a cada dois dias. Os indivíduos capturados foram identificados, marcados com brincos metálicos numerados (MONTEIRO-FILHO \& GRAIPEL, 2006) colocados na orelha esquerda quando machos e orelha direita quando fêmea para facilitar a identificação na observação do animal. Cada registro foi anotado obtendo-se as seguintes informações: data de captura, tipo de habitat, número da estação de captura, tipo de armadilha, espécie, número de cadastramento individual do animal, determinação do sexo, estágio reprodutivo, aspecto geral do corpo, dimensões corporais e comportamento após soltura. Após a obtenção dos dados biométricos, os animais foram soltos próximos as grades de captura.

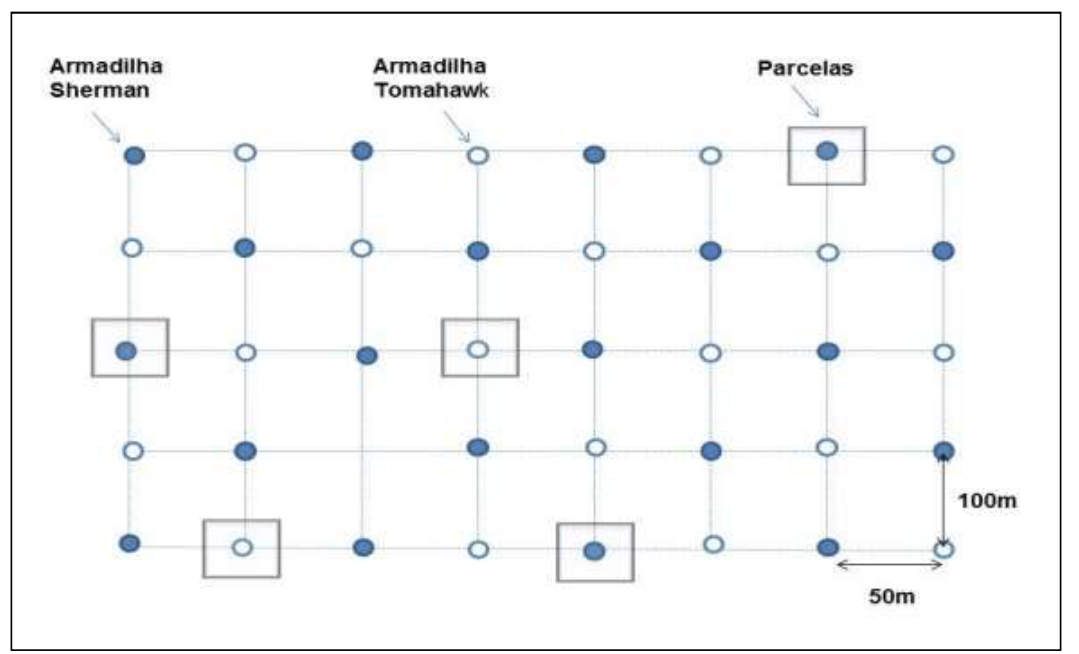

Gráfico 2: Modelo esquemático de uma grade fixa de captura de pequenos mamíferos na área de estudo. Círculos representam as estações de captura, sendo os círculos fechados as armadilhas Sherman e os abertos as armadilhas Tomahawk e os quadrados representam as parcelas de amostragem fitofisionomica.

Durante o período de amostragem foram totalizadas 69 capturas de 73 indivíduos (capturas e recapturas) distribuídos em duas ordens, três famílias e seis espécies. Com um esforço total de captura de 3.000 armadilhas-noite. O sucesso de captura para a Serra do Livramento foi de $2,43 \%$ variando entre as grades. A grade I obteve um sucesso de $4,9 \%$, a grade II de $1,3 \%$ e a grade III de 1,1\%. O número de espécies (riqueza) variou entre as grades, sendo registradas cinco espécies na grade I (45\%), três na grade II (27\%) e três na grade III (27\%).

A proporção de cada uma das espécies amostradas diferiu entre as grades, sendo Thrichomys apereoides a espécie mais abundante da grade I e II enquanto que Wiedomys 
pyrrhorrhinus foi a espécie mais abundante da grade III (Quadro 1). Ao se analisar apenas o número de indivíduos, observou-se que Thrichomys apereoides $(44,93 \%)$ foi a espécie dominante na Serra do Livramento seguido de Wiedomys pyrrhorhinus com 27,54\%. Gracilinanus agilis e Monodelphis domestica ocorreram exclusivamente na grade I e Calomys expulsus exclusivamente na grade III.

De hábito generalista e terrestre, Thrichomys apereoides foi a espécie de roedor mais comum e mais abundante, uma vez que foi capturada em todos os ambientes, em todos os períodos amostrais e apresentou o maior número de indivíduos capturados. Wiedomys pyrrhorrhynus também foi amostrado nas três grades do estudo, mas não apresentou preferência clara entre as grades.

Quadro 1: Espécies, Número de indivíduos capturados ( $N_{i c}$ e Número de indivíduos recapturados ( $N_{\text {ir }}$ ) nas grades de amostragem na Serra do Livramento, no período de dezembro de 2013 a maio de 2014.

\begin{tabular}{|c|c|c|c|c|c|c|c|c|}
\hline \multirow[t]{2}{*}{ Espécies } & \multicolumn{2}{|c|}{ Grade I } & \multicolumn{2}{|c|}{ Grade II } & \multicolumn{2}{|c|}{ Grade III } & \multicolumn{2}{|c|}{ Total } \\
\hline & Nic & Nir & $\mathrm{Nic}$ & $\mathrm{Nir}$ & $\mathrm{Nic}$ & $\mathrm{Nir}_{\mathrm{ir}}$ & $\mathrm{Nic}$ & Nir \\
\hline Thrichomys apereoides (Lund, 1839) & 24 & 2 & 6 & 0 & 1 & 0 & 31 & 2 \\
\hline Wiedomys pyrrhorhinus (Wied-Neuwied, 1821) & 7 & 1 & 5 & 0 & 7 & 1 & 19 & 2 \\
\hline Calomys expulsus (Waterhouse,1837) & 0 & 0 & 0 & 0 & 2 & 0 & 2 & 0 \\
\hline Didelphis albiventris (Lund, 1840) & 6 & 4 & 2 & 0 & 0 & 0 & 8 & 4 \\
\hline Monodelphis domestica (Wagner,1842) & 8 & 2 & 0 & 0 & 0 & 0 & 8 & 2 \\
\hline Gracilinanus agilis (Burmeister, 1854) & 1 & 0 & 0 & 0 & 0 & 0 & 1 & 0 \\
\hline Total & 46 & 9 & 13 & 0 & 10 & 1 & 69 & 10 \\
\hline
\end{tabular}

O estimador de riqueza de Jackknife $(\hat{S})$ indicou que a comunidade de pequenos mamíferos não voadores da Serra do Livramento possui sete espécies e a riqueza de espécies pode variar entre cinco e nove espécies com um intervalo de confiança de $95 \%\left(t_{\alpha}=4,303\right)$. A diversidade de pequenos mamíferos obtida para a grade I na Serra do Livramento foi superior ao observado para as demais grades amostradas para o índice de Shannon-Wiener, enquanto que para o índice de Simpson a grade III foi a que apresentou a maior diversidade de espécies (Tabela 2).

Tabela 2: Índices de Diversidade de Shannon-Wiener $\left(H^{\prime}\right)$, de Simpson $(1-D)$ e índice de Equitabilidade de Simpson $\left(E_{1 / \widehat{D}}\right)$ para pequenos mamíferos nas três grades amostrais analisadas na Serra do Livramento.

\begin{tabular}{llll}
\hline Parâmetros & $H^{\prime}$ & $1-D$ & $E_{1 / \widehat{D}}$ \\
Áreas & 1,28 & 0,33 & \\
\hline Grade I & 1,01 & 0,33 & \\
Grade II & 0,80 & 0,49 & \\
Grade III & 1,864 & 0,686 & 0,545 \\
Serra do Livramento & \\
\hline
\end{tabular}

O índice de similaridade de Morisita, baseado na abundância e diversidade, indicou similaridade elevada entre as grades I e $\|\left(I_{m}=0,878\right)$ evidenciando que as comunidades de pequenos mamíferos destas áreas são muito semelhantes. A similaridade foi mediana entre as grade II e III ( $\left.I_{m}=0,682\right)$ e baixa entre as grades I e III ( $\left.I_{m}=0,359\right)$ (Gráfico 3). A baixa 
similaridade entre as grades I e III decorre da exclusividade de Monodelphis domestica e Gracilinanus agilis na grade I e de Calomys expulsus na grade III (Gráfico 3).

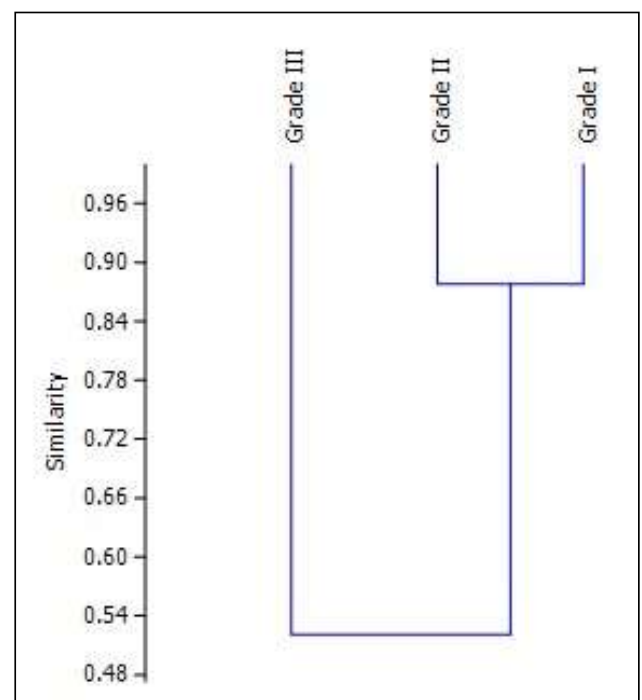

Gráfico 3: Dendrograma de dos índices similaridade de Morisita entre as grades amostrais da Serra do Livramento.

No intuito de verificar essa correlação, foi realizada uma analise de correspondência canônica que buscou correlacionar alguns parâmetros fitossociológicos com a ocorrência de pequenos mamíferos em cada uma das grades amostradas. No diagrama de ordenação (Gráfico 4) os eixos da análise de correspondência canônica representam a contribuição relativa de cada componente para explicar a variação total dos dados. As espécies de pequenos mamíferos estão representadas por pontos ótimos no espaço bidimensional, enquanto que os parâmetros fitofisionômicos (altura média, densidade, frequência total e dominância) e as famílias vegetais mais representativas, aparecem como vetores, indicando a direção das mudanças destas variáveis no espaço de ordenação.

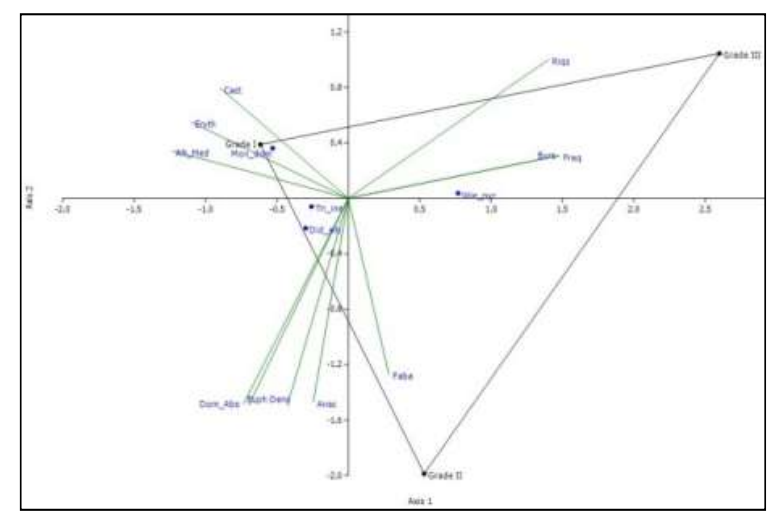

Gráfico 4: Representação gráfica da análise de correspondência canônica (CCA) comparando a matriz de características ambientais com a matriz de abundância de pequenos mamíferos não-voadores da Serra do Livramento, Pernambuco. Vetores correspondem às variáveis ambientais: Anac - Anacardiaceae; Burs - Burseraceae; Cact - Cactaceae; Euph - Euphorbiaceae; Eryth - Eruthroxilaceae; Faba - Fabaceae; Riqz - Riqueza; Alt_Med = Altura média; Dens - Densidade; Dom_Abs - Dominância Absoluta; Freq - Frequencia; círculos azuis correspondem as espécies: Cal_exp - Calomys expulsus; Did_alb-Didelphis albiventris; Gra-agi - Gracilinanus agilis; Mon_dom - Monodelphis domestica; Tri_ape - Trychomys apereoides; Wie-Pyr - Wiedomys pyrrorhinus; e os círculos pretos correspondem as grades de amostragens.

O primeiro eixo da ordenação explicou $88,6 \%$ da variação total na abundancia de pequenos mamíferos e o segundo eixo apenas $11,4 \%$. No eixo 1 as variáveis mais positivas importantes foram riqueza $(0,973)$, frequência absoluta $(0,957)$, presença de Burseraceae $(0,957)$ 
e a variável negativa mais importante foi a altura média $(-0,745)$. No eixo 2 as variáveis negativas mais importantes foram presença de Anacardiaceae $(-0,999)$, densidade $(-0,994)$, dominância absoluta $(-0,598)$ enquanto que a presença de Cactaceae $(0,513)$ foi a variável positiva mais importante (Tabela 3).

Tabela 3: Correlação entre as variáveis ambientais de (Serra do Livramento, PE) e os eixos de ordenação para cada uma das variáveis obtidas pela análise de correspondência canônica.

\begin{tabular}{lll}
\hline & Eixo 1 & Eixo 2 \\
\hline Auto valor & 0,362 & 0,046 \\
Variância da abundância de espécies (\%) & 88,6 & 11,4 \\
Correlação das variáveis ambientais & & $-0,9998^{*}$ \\
Anacarciaceae & $-0,288$ & 0,0152 \\
Burseraceae & $0,9576^{*}$ & $0,6823^{*}$ \\
Cactaceae & $-0,4902$ & $-0,9543^{*}$ \\
Euphorbiaceae & $-0,5736$ & 0,5379 \\
Erythroxylaceae & $-0,6406$ & $-0,9325$ \\
Fabaceae & 0,0619 & 0,5131 \\
Riqueza & $0,9733^{*}$ & 0,4105 \\
Altura Média & $-0,7448^{*}$ & $-0,9939^{*}$ \\
Densidade & $-0,4053$ & $-0,9449^{*}$ \\
Dominância Absoluta & $-0,5979$ & 0,0152 \\
Frequência Absoluta & $0,9576^{*}$ & \\
\hline
\end{tabular}

*variáveis que mais contribuem para a variação dos dados.

Verificou-se que as variáveis Anacardiaceae (ANAC), Euphorbiaceae (EUPH), Densidade (DENS), Dominância Absoluta (DOM_ABS) contribuíram para a ocorrência de Didelphis albiventris e Trichomys apereoides na grade I, espécies generalistas e de ocorrência em ambientes com algum grau de perturbação (FONSECA \& ROBISON, 1990; BONVICINO et al., 2002; VIVEIROS DE CASTRO \& FERNANDEZ, 2004; GHELER-COSTA, 2006). Foram registradas na grade I quatro das sete espécies da família Euphorbiaceae presentes na Serra do Livramento, destas Croton blanchetianus ( $\mathrm{n}=82$ ) destacando-se das demais em relação a abundancia. A ocorrência de Monodelphis domestica na grade I, esteve relacionada a características ambientais distintas daquelas percebidas por Didelphis albiventris e Trichomys apereoides. Neste estudo, a grade II apresentou maior densidade e dominância absoluta, e isso pode ser reflexo do quanto dessa área foi alterada, proporcionando espaços e clareiras que auxiliam no aumento da germinação de espécies pioneiras.

A grade III diferencia-se das demais em relação as variáveis riqueza, frequência absoluta e presença de Burseraceae (Commiphora leptophloeos). Não houve preferência por essa grade de amostragem por nenhuma das espécies com mais de cinco amostragens, entretanto deve-se destacar a ocorrência de Calomys expulsus exclusivamente na grade III. O sucesso de captura de pequenos mamíferos na Serra do Livramento $(2,43 \%)$ foi satisfatório se comparado com o sucesso de captura de comunidades de pequenos mamíferos não voadores obtidos em outros estudos em áreas de Caatinga (BEZERRA et al., 2014; FREITAS et al., 2005). Esse resultado pode ser o reflexo da heterogeneidade ambiental presente na Serra do Livramento.

A riqueza registrada para a Serra do Livramento $(n=6)$ foi similar a obtida em estudos de comparação de pequenos mamíferos em na Caatinga (FREITAS et al., 2005; NASCIMENTO et al., 2013) e inferior se comparado a estudos em outros áreas abertas de Cerrado e Mata de 
restinga (RIBEIRO \& MARINHO-FILHO, 2005; GRAIPEL et al., 2006; CÁCERES et al., 2008; QUINTELA et al., 2012, LAYME et al., 2012). A baixa riqueza de espécies registrada para a Serra do Livramento pode ser um reflexo da menor complexidade estrutural dos ambientes abertos, quando comparados a ambientes florestados (AUGUST, 1983; FONSECA, 1989; KERLEY, 1992; LYRA JORGE et al., 2001) podendo a riqueza da Serra do Livramento estar relacionada a estrutura da vegetação, ao tipo de solo existente ou ainda ser o reflexo de uma amostragem realizada somente em um período sazonal.

A diversidade de pequenos mamíferos encontrada na Serra do Livramento foi satisfatória em relação aos estudos relacionando pequenos mamíferos (LYRA JORGE et al., 2001; RIBEIRO \& MARINHO-FILHO,2005), mas inferior aquela obtida por Pinto et al. (2009). O principal fator que pode interferir no aumento da diversidade é a composição do habitat, pois permite a ocorrência de espécies animais com diferentes hábitos. Segundo Alho (1981) a densidade da vegetação interfere na diversidade das espécies de pequenos mamíferos onde, a diversidade é menor em áreas mais abertas e maiores em áreas densas. Alho (1981), Lathman e Ricklefs (1993), MarinhoFilho et al. (1994), Bonvicino et al. (2002) apontam que em comunidades tropicais, a riqueza e a diversidade de espécies aumentam com a intensificação da complexidade da estrutura da vegetação, portanto, quanto maior a complexidade vegetacional maior o número de espécies naquele habitat. Lyra Jorge et al. (2001), Ribeiro e Marinho (2005) sugerem que a estrutura das comunidades de pequenos mamíferos pode estar diretamente interligada com a estrutura fitofisionômica dos ambientes amostrados.

A presença de espécies da família Euphorbiaceae, em especial do elevado numero de indivíduos de Croton blanchetianus (grade I) e Croton heliotropiifolius ( $\mathrm{n}=104)$ na grade II, sugerem que nestas grades há porções com certo grau de perturbação, visto que as espécies do gênero Croton são pioneiras, pois tem como características a facilidade de reprodução e dispersão (SAMPAIO et al., 1998; PEREIRA et al., 2001; SILVA et al., 2012). Para Monodelphis domestica as características ambientais de maior relevância foram a presença de Erythroxylum sp. $(n=16)$, Cactaceae e a altura média da vegetação $(3,73 m)$. A ocorrência de Erythroxylum sp. pode ser considerada como um indicativo de bom estágio de conservação da vegetação, por se tratar de um gênero com espécies de estágios seriais secundários iniciais e tardios (QUEIROZ et al., 2006). A densidade e dominância baixas da grade I pode interferir na ocorrência de Monodelphis domestica e a altura média superior da grade II pode ser um elemento importante na ocorrência de Gracilinanus agilis, visto que esta espécie apresenta hábitos arbóreos.

A grade III diferencia-se das demais por apresentar a maior diversidade e equitabilidade florística, e um maior número de espécies secundárias e tardias para a Caatinga (Sapium glandulosum, Anadenathera colubrina, Amburana cearensis, Schinopsis brasiliensis, Auxemma oncocalyx) o que pode explicar a baixa similaridade da comunidade de pequenos mamíferos desta grade com as demais grades amostradas. Wiedomys pyrrorhinus não apresentou preferência clara por nenhuma as grades estudadas. As variações observadas na estrutura das comunidades de pequenos mamíferos podem ser elucidadas com base na estrutura das comunidades vegetais, 
que por sua vez são influenciadas por aspectos físicos do ambiente como tipo de solos, variações altitudinais e ações antrópicas.

\section{CONCLUSÕES}

Conclui-se que a Serra do Livramento apresenta heterogeneidade ambiental visto que se pode diferenciar fito fisionomicamente das três grades de amostragem de pequenos mamíferos. Essa diferença na composição florística e fitofisionomia das grades amostrais contribuiu para as diferenças na composição (riqueza, diversidade e similaridade) das comunidades de pequenos mamíferos presentes nas grades amostrais. Embora, este estudo não tenha contemplado um período sazonal completo, os dados obtidos são relevantes e podem contribuir para o estabelecimento de estratégias de conservação da Serra do Livramento no estado do Pernambuco, principalmente por essa área sofrer pressão de caça, desmatamento e estar na área de influência diretamente afetada da maior obra de construção civil do nordeste Brasileiro, o Projeto de Integração do Rio São Francisco com as Bacias Hidrográficas do Nordeste Setentrional.

\section{REFERÊNCIAS}

ALHO, C. J. R.. Small mammals populations of Brazilian Cerrado: the dependence of abundance and diversity on habitat complexity. Revista Brasileira de Biologia, v.41, p.223-230, 1981.

AUGUST, P.V.. The role of habitat complexity and heterogeneity in structuring tropical mammal communities. Ecology, v.64, n.6, p.1495-1507, 1983.

BEZERRA, A. M. R.; BONVICINO, C.; CUNHA, A. S.. Subsidies a poorly knon endemic semiarid biome of Brazil: non-volant mammals of na eastern region of Caatinga. Zoological Studies. v.53, n.16, p.1-13, 2014.

BONVICINO, C. R.; LINDBERGH, S. M.; MAROJA, L. S.. Small Non-Flying Mammals From Conserved And Altered Areas Of Atlantic Forest And Cerrado: Comments On Their Potential Use For Monitoring Environment. Brazilian Journal of Biology. São Carlos, v.62, n.4, p.765-774, 2002.

CÁCERES, N. C.; MONTEIRO-FILHO, E. L. A.. Population dynamics of the common opossum, Didelphis marsupialis (Mammalia, Marsupialia) in southern Brazil. International Journal of Mammalian Biology, v. 63, p.169-172. 1998.

CACERES, N. C.; CASELLA, J.; VARGAS, C. F.; PRATES, L. Z.; TOMBINI, A. A. M.; GOULART, C. S.; LOPES, W. H.. Distribuiçao geográfica de pequenos mamíferos não voadores nas bacias dos Rios Araguaia e Paraná, região centro-sul do Brasil. Iheringia. Série Zoologia, v. 98, p.1-8, 2008.

FONSECA, G. A. B.; ROBINSON, J. G.. Forest size and structure: competitive and predatory effects on small mammal communities. Biological Conservation, v.53, p.265-294,1990.

FORERO-MEDINA, G. M.; VIEIRA, M. V.. Conectividade funcional e a importância da interação organismopaisagem. Oecologia Brasiliense, v.11, n.4, p.493-502. 2007.

FREITAS, R. R.; ROCHA, L. B. R.; SIMÕES-LOPES, P. C.. Habitat structure and small abundances in one semiarid landscape in the Brazilian Caatinga. Revista Brasileira de Zoologia. v.22, n.1, p.119-129, 2005.

GHELER-COSTA, C.. Distribuição e abundância de pequenos mamíferos em relação à paisagem da acia do Rio Passa-Cinco, São Paulo, Brasil. Tese (Doutorado) - Universidade de São Paulo, Piracicaba. 2006. 
GRAIPEL, M. E.;CHEREM, J. J.; MONTEIRO-FILHO, E. L. A.; GLOCK, L.. Dinâmica populacional de marsupiais e roedores no Parque Municipal da Lagoa do Peri, Ilha de Santa Catarina, Sul do Brasil. Mastozoologia Neotropical. v.13, n.1, p.31-49. 2006.

LATHAM, R. E.; RICKLEFS, R. E.. Global patterns of tree scpecies richness in moist forest: energy-diversity theory does not account for variation in species richness. Oikos, v.67, n.2, p.325-333. 1993.

LAYME, V. M. G.; CANDELÁRIA, L. P.; SANTOS, A. P. M. P.; SILVA, P. B. A.. Estrutura da comunidade de pequenos mamíferos não voadores em campos nativos do pantanal de Poconé. Oecologia Australis, v.16, p. 949-957, 2012.

LYRA JORGE, M. C.; PIVELLO, V. R.; MEIRELLES, S. T.; VIVO, M.. Riqueza e abundância de pequenos mamíferos em ambientes de Cerrado e Floresta, na Reserva Cerrado do Pé-de-gigante, Parque Estadual de Vassununga (Santa Rita do Passa Quatro, SP). Naturalia, v.26, p.287-302. 2001.

MARINHO-FILHO, J.; REIS, M. L.; OLIVEIRA, P. S.; VIEIRA, E. M.; PAES, M. N.. Diversity Standards and small mammal numbers: conservation of the Cerrado Biodiversity. Academia Brasileira de Ciências, v.66, p.149-157, 1994.

MONTEIRO - FILHO, E. L. A.; GRAIPEL, M. E.. Captura e marcação. Campo Grande: Universidade Federal do Mato Grosso do Sul, 2006.

NASCIMENTO, A. L. C. P.; FERREIRA, J. D. C.; MOURA, G. J. B.. Marsupiais de uma área de Caatinga (Pernambuco, Brasil) com registro de nova localidade para Caluromys philander (Linnaeus, 1758). Revista Iber-Americana de Ciências Ambientais. Aquibadã, v.4, n.1, p.105-110, 2013. DOI:

http://dx.doi.org/10.6008\%2FESS2179-6858.2013.001.0008

PAGLIA, A. P.; MARCOS JUNIOR, P.; COSTA, F. M.; PEREIRA, R. F. E.; LESSA, G.. Heterogeneidade estrutural e diversidade de pequenos mamíferos em um fragmento de mata secundária de Minas Gerais, Brasil. Revista Brasileira de Zoologia, v.12, n.1, p.67-69, 1995.

PEREIRA, I. M.; ANDRADE, L. A.; COSTA, J. R. M.; DIAS, J. M.. Regeneração natural em um remanescente de caatinga sob diferentes níveis de perturbação, no Agreste Paraibano. Acta Botanica Brasilica, v.15, n 3, p.413-426, 2001.

PINTO, I. S.; LOSS, A. C. C.; FALQUETO, A.; LEITE, Y. L. R.. Pequenos mamíferos não voadores em fragmentos de Mata Atlântica e áreas agrícolas em Viana, Espírito Santo, Brasil. Biota Neotropica. v.9, n.3, p.355-360, 2009.

QUEIROZ, J. A.; TROVÃO, D. M .B.; OLIVEIRA, A. B.; OLIVEIRA, E. C. S.. Análise da Estrutura Fitossociológica da Serra do Monte, Boqueirão, Paraíba. Revista de Biologia e Ciências da Terra, v.6, n. 1, p.251-259. 2006.

QUINTELA, F. M.; SANTOS, M. B.; CHRISTOFF, A. U.;GAVA, A.. Pequenos mamíferos não-voadores (Didelphimorphia, Rodentia) em dois fragmentos de mata de restinga de Rio Grande, Planície Costeira do Rio Grande do Sul. Biota Neotropica, v.12, n.1, p.261-266, 2012.

REVILLA, E.; WIEGAND, T.. Individual movement behavior, matrix heterogeneity, and the dynamics of spatially structured populations. Proceedings of the National Academy of Sciences of the USA, v.105, n. 49, p.19120 - 19125, 2008.

REVILLA, E.; WIEGAND, T.; PALOMARES, F.; FERRERAS, P.; DELIBES, M.. Effects of matrix heterogeneity on animal dispersal: from individual behavior to metapopulation-level parameters. The American naturalist, v.164, n.5, p130-153, 2004.

RIBEIRO, R.; MARINHO-FILHO, J.. Estrutura da comunidade de pequenos mamíferos (Mammalia, Rodentia) da estação Ecológica de Águas Emendadas, Planaltina, Distrito Federal, Brasil. Revista Brasileira de Zoologia, v. 22, n. 4, p.898-1007, 2005.

SAMPAIO, E. V. S. B.; ARAUJO, E. L.; SALCEDO, I. H.; TIESSEN, H.. Regeneração da vegetação de caatinga após corte e queima, em Serra Talhada (PE). Pesquisa Agropecuária Brasileira, Brasília. v.33, p. 621-632, 1998 
SILVA, S. O.; FERREIRA, R. L. C.; SILVA, J. A. A.; LIRA, M. A.; ALVES JUNIOR, F. T. A.; CANO, M. O. O.; TORRES, J. E. L.. Regeneração natural em um remanescente de Caatinga com diferentes históricos de uso no agreste pernambucano. Revista Árvore, Viçosa, v.36, n.3, p.441-450, 2012.

TABARELLI, M.; SILVA, J. M. C.. Áreas de ações prioritárias para a conservação da biodiversidade da Caatinga. In: Leal, R.I, Silva, J. M.C (Org.). Ecologia da Conservação da Caatinga. p.781-800, 2003.

VIVEIROS DE CASTRO, E. B.; FERNANDEZ, F. A. S.. Determinants of differential extinction vulnerabilities of small mammals in Atlantic forest fragments in Brazil. Biological Conservation. v.119, p.73-80, 2004. 